\author{
ANNALES \\ POLONICI MATHEMATICI \\ XXVI (1972)
}

\title{
On superposition of quasianalytic functions
}

\author{
by W. Pleśniak (Kraków)
}

Let $E$ be a compact set in the space $C^{n}$ of $n$ complex variable$z=\left(z_{1}, \ldots, z_{n}\right)$. Let $\mathscr{C}(E)$ denote the Banach algebra of all complex funes tions continuous in $E$ with the norm

$$
\|f\|_{E}=\max _{z \in E}|f(z)|, \quad f \in \mathscr{C}(E) .
$$

Denote by $\mathscr{E}_{v}(f, E)$ the $v$-th measure of the best Cebysev approximation to $f \in \mathscr{C}(E)$ on $E$ by polynomials in $z$, i.e.

$$
\mathscr{E}_{v}(f, E)=\inf \left\|f-P_{v}\right\|_{E},
$$

where inf is spread over all the polynomials $P_{v}$ of degree $\leqslant \nu$. Let $\mathscr{B}(E)$ denote the subset of $\mathscr{C}(E)$ consisting of all the functions satisfying the following condition:

$$
\liminf _{v \rightarrow \infty} \sqrt[v]{\mathscr{E}_{v}(f, E)}<1 .
$$

The functions of $\mathscr{B}(E)$ are called quasianalytic on $E$ in Bernstein's sense. In the case $n=1$, if $E$ is a compact interval of the real axis $R$, the basic properties of functions $f \in \mathscr{B}(E)$ may be found in [1] or [6]. The term "quasianalytic" arises from the following identity principle given by Bernstein:

If $E$ and $I$ are compact intervals in $R$ and $I \subset E$, then every function $f \epsilon \mathscr{B}(E)$ vanishing on $I$ is identically equal to zero.

In the case $n \geqslant 1$, a generalization of this result has been given in [4].

Let $\left\{v_{k}\right\}$ be an increasing sequence of positive integers. Denote by $\left[\left\{v_{k}\right\}\right]$ the set of all increasing sequences $\left\{\mu_{k}\right\}$ of positive integers such that $1 / M<\mu_{k} / v_{k}<M$ for $k \geqslant 1, M$ being a positive constant dependent on $\left\{\mu_{k}\right\}$. Denote by $\mathscr{B}\left(E,\left\{\nu_{k}\right\}\right)$ the set of all functions $f \in \mathscr{B}(E)$ such that

$$
\lim _{k \rightarrow \infty} \sqrt[v_{k}]{\mathscr{E}_{v_{k}}(f, E)}<1
$$

and define $\mathscr{B}\left(E,\left[\left\{\nu_{k}\right\}\right]\right)=\left\{f \in \mathscr{B}\left(E,\left\{\mu_{k}\right\}\right):\left\{\mu_{k}\right\} \in\left[\left\{v_{k}\right\}\right]\right\}$. One can check that $\mathscr{B}\left(E,\left[\left\{v_{k}\right\}\right]\right)$ is a ring with respect to the ordinary pointwise addi- 
tion and multiplication of functions. If $E$ satisfies the assumptions of the identity theorem in [4], then the ring $\mathscr{B}\left(E,\left[\left\{v_{k}\right\}\right]\right)$ is a domain of integrity.

The main purpose of this paper is to answer in the negative the following natural question:

(I) Let $f \in \mathscr{B}\left(E,\left[\left\{v_{k}\right\}\right]\right)$ and $g \in \mathscr{B}\left(f(E),\left[\left\{v_{k}\right\}\right]\right)$. Does this imply that $g \circ f \in \mathscr{B}\left(E,\left[\left\{v_{k}\right\}\right]\right)$ ?

It follows from a result of Siciak (cf. Lemma 1) that every function $\varphi$ holomorphic in a neighbourhood of a polynomially convex compact set $F$ in $C^{n}$ is a member of $\mathscr{B}\left(F,\left\{v_{k}\right\}\right)$ for any $\left\{v_{k}\right\}$. Hence, in order to answer question (I) we may consider the following problem:

Let $f \in \mathscr{B}\left(E,\left[\left\{v_{k}\right\}\right]\right)$ and let $\varphi$ be a holomorphic function in a neighbourhood of $f(E)$. What conditions are necessary and sufficient for $\varphi \circ f \in \mathscr{B}\left(E,\left[\left\{\boldsymbol{v}_{k}\right\}\right]\right)$ ?

If $E$ is polynomially convex, we give some sufficient condition for (II). It is also a necessary one if $\varphi$ is rational and the extremal function $\Phi(z, E)$ (introduced by J. Siciak in [5]) is continuous in $E$ (see Theorem 1). On the other hand, given $f \in \mathscr{B}\left(E,\left[\left\{v_{k}\right\}\right]\right)$, we may always assume that $E$ is polynomially convex (see Lemma 3). Hence, Counter-examples 1, 2 and 3 give us a negative reply to (I).

Nevertheless, in accordance with Examples 4 and 5, there exist functions $f \in \mathscr{B}\left(E,\left[\left\{v_{k}\right\}\right]\right)$ and $g \in \mathscr{B}\left(f(E),\left[\left\{v_{k}\right\}\right]\right), g(w) \not \equiv w$, such that $g \circ f_{\epsilon} \mathscr{B}\left(E,\left[\left\{v_{k}\right\}\right]\right)$.

Finally, we note that a simple characterization of essentially quasianalytic functions on $E$ (i.e. functions not continuable to holomorphic functions in any neighbourhood of $E$ ) is given by Lemma 2 . In the case $n=1$, because of the Montel theorem, that lemma can be formulated as follows.

$A$ complex function $f$ defined and bounded on a compact set $E$ is the restriction to $E$ of a function $F$ holomorphic in a neighbourhood of the polynomially convex envelope $\hat{E}$ of $E$ if and only if there exist polynomials $\left\{\boldsymbol{P}_{k}\right\}$, an open set $U, E \subset U$, and at least two distinct points $a, b \in C$ such that

and

$$
\lim _{k \rightarrow \infty}\left\|f-P_{k}\right\|_{E}=0
$$

$$
P_{k}(U) \subset C \backslash\{a, b\}, \quad k \geqslant 1 .
$$

We start from some lemmas. The first one is a slight modification of the well-known result of Siciak [5].

LEMMA 1. Let $\left\{f_{k}\right\}$ be a sequence of bounded holomorphic functions in an open set $\Omega$ in $C^{n}$. Write $M_{k}=\sup _{z \in \Omega}\left|f_{k}(z)\right|$. Then for every polynomially 
convex compact set $E, E \subset \Omega$, there exist positive constants $M$ and $\varrho, \varrho \in(0,1)$, independent of $k$ and such that

$$
\mathscr{E}_{\nu}\left(f_{k}, E\right) \leqslant M M_{k} \varrho^{\nu}, \quad \nu \geqslant 1, k \geqslant 1
$$

Proof. Fix a polynomially convex compact set $E, E \subset \Omega$, and a number $R>1$. It follows from the definition of the polynomial convexity and from the Borel-Lebesgue theorem that there exist polynomials $P_{1}, \ldots, P_{m}$ such that $\left\|P_{j}\right\|_{E} \leqslant 1(j=1, \ldots, m)$ and

$$
E \subset \text { int } L \subset L=\left\{z \in C^{n}:\left|P_{j}(z)\right| \leqslant R, j=1, \ldots, m\right\} \subset \Omega .
$$

Put $g_{k}=f_{k} / M_{k}$. Applying the Weil integral formula in $L$, by the same reasoning as in [5], p. 345, for every $g_{k}$ we find a sequence of polynomials $\left\{Q_{v}^{k}\right\}_{v \geqslant 1}, \operatorname{deg} Q_{v}^{k} \leqslant v$, such that

$$
\left\|g_{k}-Q_{\nu}^{k}\right\|_{E} \leqslant M \varrho^{\nu}, \quad \nu \geqslant 1,
$$

where the constants $M$ and $\varrho$ are independent of $k, \varrho \epsilon(0,1)$. Hence putting $R_{v}^{k}=M_{k} Q_{v}^{k}$ gives

$$
\left\|f_{k}-R_{\nu}^{k}\right\|_{E} \leqslant M M_{k} \varrho^{\nu}, \quad \nu \geqslant 1,
$$

for $k=1,2, \ldots$ The proof is completed.

Given a compact set $E$ in $C^{n}$, we shall denote by $\hat{E}$ the polynomially convex envelope of $E$. By Lemma 1 (for $f_{k}=f$ ) one can easily prove the following

LEMma 2. Let $f$ be a complex function defined and bounded on $\boldsymbol{E}$. $A$ necessary and sufficient condition that $f$ be the restriction to $E$ of a function $\tilde{f}$ holomorphic in a neighbourhood of $\hat{E}$ is that there exist polynomials $\left\{P_{k}\right\}$ and an open set $U, \hat{E} \subset U$, such that

$$
\lim _{k \rightarrow \infty}\left\|f-P_{k}\right\|_{E}=0
$$

and the sequence $\left\{\boldsymbol{P}_{k}\right\}$ forms a normal family in $U$.

LEMма 3. Suppose that $f \in \mathscr{B}\left(E,\left\{v_{k}\right\}\right)$. Then there exist a function $\tilde{f} \in \mathscr{B}\left(\hat{E},\left\{v_{k}\right\}\right)$ such that $\left.\tilde{f}\right|_{E}=f$.

Proof. Take polynomials $\left\{P_{v_{k}}\right\}, \operatorname{deg} P_{p_{k}} \leqslant v_{k}$, such that

$$
\left\|f-P_{v_{k}}\right\|_{E} \leqslant M \varrho^{v k}, \quad k \geqslant 1,
$$

where $M$ and $\varrho$ are constants independent of $k, \varrho \epsilon(0,1)$. Because of $(1)$ and the triangle inequality, the function $f$ can be expanded into the series

$$
f(z)=P_{v_{1}}(z)+\sum_{k=1}^{\infty}\left[P_{v_{k+1}}(z)-P_{v_{k}}(z)\right]
$$


convergent uniformly in $E$. By the definition of $\hat{E}$ series (2) is uniformly convergent in $\hat{E}$ to a function $\tilde{f}$. Moreover,

$$
\begin{aligned}
\left\|\tilde{f}-P_{v_{k}}\right\|_{\hat{E}} & =\left\|\sum_{l=k}^{\infty}\left(P_{v_{l+1}}-P_{\nu_{l}}\right)\right\|_{\hat{E}} \leqslant \sum_{l=k}^{\infty}\left\|P_{v_{l+1}}-P_{v_{l}}\right\|_{E} \\
& \leqslant M \sum_{l=k}^{\infty}\left(\varrho^{v_{l+1}}+\varrho^{v_{l}}\right) \leqslant \frac{2 M}{1-\varrho} \varrho^{v_{k}} .
\end{aligned}
$$

This implies that $\tilde{f} \epsilon \mathscr{B}\left(\hat{E},\left\{\nu_{k}\right\}\right)$ as asserted.

LeMma 4. Let $E$ be a compact set in $C^{n}$ and let $\mathscr{F}$ be a family of polynomials satisfying the following conditions:

(i) $|f(z)| \geqslant m>0, z \in E, f \in \mathscr{F}$,

(ii) there exists an open set $U$ in $C^{n}$ such that $E \subset U$ and $f(z) \neq 0$ for $z \in U, f \in \mathscr{F}$.

Then, for every $\omega>1$ there exists an open set $V$ in $C^{n}$ such that $E \subset V$ and

$$
|f(z)| \omega^{\operatorname{deg} t} \geqslant m, \quad z \epsilon V, \quad f \in \mathscr{F}\left({ }^{1}\right) .
$$

Proof. Fix a number $\omega>1$ and a point $a=\left(a_{1}, \ldots, a_{n}\right) \in E$ and put $\theta=\omega^{-1 / n}$. Given a polynomial $f \in \mathscr{F}$, we write

$$
g\left(z_{1}\right)=f\left(z_{1}, a_{2}, \ldots, a_{n}\right)=\beta\left(z_{1}-a_{1}\right) \ldots\left(z_{1}-a_{1}\right),
$$

where the numbers $\beta, \alpha_{j}(j=1, \ldots, l)$ may depend on $f$ and on the point $a, 0 \leqslant l \leqslant \operatorname{deg} f$. By assumption (i) we obtain

$$
|\beta| \geqslant \frac{m}{\left|a_{1}-a_{1}\right| \ldots\left|a_{1}-\alpha_{l}\right|} .
$$

Hence

$$
\left|g\left(z_{1}\right)\right| \geqslant m\left|\frac{z_{1}-a_{1}}{a_{1}-a_{1}}\right| \ldots\left|\frac{z_{1}-a_{l}}{a_{1}-a_{l}}\right| .
$$

Take $\delta=\operatorname{dist}\left(E, C^{n} \backslash U\right) / 2 \sqrt{n}$. By (ii), $f(z) \neq 0$ for $z \in P(a, \delta)$ $=K\left(a_{1}, \delta\right) \times \ldots \times K\left(a_{n}, \delta\right)$, where $K\left(a_{k}, \delta\right)=\left\{z_{k} \epsilon C:\left|z_{k}-a_{k}\right| \leqslant \delta\right\}$. Hence $\left|a_{1}-a_{j}\right|>\delta$ for $j=1, \ldots, l$. So, setting $\delta_{\omega}=(1-\theta) \delta$ gives

$$
\left|\frac{z_{1}-a_{j}}{a_{1}-a_{j}}\right| \geqslant 1-\left|\frac{a_{1}-z_{1}}{a_{1}-a_{j}}\right| \geqslant 1-\frac{\delta_{\omega}}{\delta}=\theta \quad \text { for } z_{1} \in K\left(a_{1}, \delta_{\omega}\right) .
$$

Hence by (1) and (3) we obtain

$$
\left|f\left(z_{1}, a_{2}, \ldots, a_{n}\right)\right| \geqslant m \theta^{l} \geqslant m \theta^{\mathrm{deg} f} \quad \text { for } z_{1} \in K\left(a_{1}, \delta_{\omega}\right) .
$$

(1) If $n=1$ and $E$ is connected, this lemma is due to Leja [2]. He proved it by means of his well-known Polynomial Lemma. Our proof is a direat one. 
By induction, in order to complete the proof it is enough to show that:

if

$$
|f(z)| \geqslant m \theta^{k \mathrm{deg} t}
$$

$$
\text { for } z \in K\left(a_{1}, \delta_{\omega}\right) \times \ldots \times K\left(a_{k}, \delta_{\omega}\right) \times a_{k+1} \times \ldots \times a_{n},
$$

then

$$
\begin{aligned}
& |f(z)| \geqslant m \theta^{(k+1) \mathrm{deg} t} \\
& \quad \text { for } z \epsilon K\left(a_{1}, \delta_{\omega}\right) \times \ldots \times K\left(a_{k+1}, \delta_{\omega}\right) \times a_{k+2} \times \ldots \times a_{n} .
\end{aligned}
$$

To this end fix a point $b^{k}=\left(b_{1}, \ldots, b_{k}\right) \in K\left(a_{1}, \delta_{\omega}\right) \times \ldots \times K\left(a_{k}, \delta_{\omega}\right)$. Then the polynomial of one variable $z_{k+1}$

$$
g\left(z_{k+1}\right)=f\left(b^{k}, z_{k+1}, a_{k+2}, \ldots, a_{n}\right)
$$

may be written in the form

$$
g\left(z_{k+1}\right)=\beta\left(z_{k+1}-\alpha_{1}\right) \ldots\left(z_{k+1}-\alpha_{l}\right),
$$

where $\beta, \alpha_{j}(j=1, \ldots, l)$ are numbers dependent on $f, b^{k}$ and $a_{k+2}, \ldots$ $\ldots, a_{n}, 0 \leqslant l \leqslant \operatorname{deg} f$. By the same reasoning as in the proof of $(5)$ one can show that

$$
\left|g\left(z_{k+1}\right)\right| \geqslant m \theta^{(k+1) \operatorname{deg} f} \quad \text { for } z_{k+1} \in K\left(a_{k+1}, \delta_{\omega}\right)
$$

independently of the choice of $b^{k} \epsilon K\left(a_{1}, \delta_{\omega}\right) \times \ldots \times K\left(a_{k}, \delta_{\omega}\right)$, which ends the proof of (6). By (5) and (6) we obtain

$$
|f(z)| \omega^{\mathrm{deg} t} \geqslant m \quad \text { for } z \in P\left(a, \delta_{\omega}\right) .
$$

Since $\delta_{\omega}$ does not depend on the choice of the point $a \epsilon E$ and $f \epsilon \mathscr{F}$, we get the assertion of the lemma with $V=\bigcup_{a \in E} \operatorname{int} P\left(a, \delta_{\omega}\right)$.

LEMMA 5. Let $f$ be a complex function defined and bounded on a compact set $E$ in $C^{n}$. Let $\phi$ be a function holomorphic in a neighbourhood $\Omega$ of the set $F=f(E)$. If for a sequence of polynomials $\left\{P_{k}\right\}$

$$
\lim _{k \rightarrow \infty}\left\|f-P_{k}\right\|_{E}=0,
$$

then there exist constants $M>0$ and $K_{0}>0$ such that

$$
\left|\varphi(f(z))-\varphi\left(P_{k}(z)\right)\right| \leqslant M\left|f(z)-P_{k}(z)\right|, \quad z \in E, k \geqslant k_{0} .
$$

Proof. Fix an $\delta, \quad 0<\delta<\operatorname{dist}(F, \partial \Omega)$. Write $\tilde{M}=\sup \{|\varphi(w)|$ : $\operatorname{dist}(w, F) \leqslant \delta\}$. By the Schwarz inequality we obtain

$$
|\varphi(w)-\varphi(b)| \leqslant \frac{2 M}{\delta}|w-b| \quad \text { for } w \in K(b, \delta / 2), b \in F .
$$


Hence, by fixing a $k_{0}$ such that $\left\|f-P_{k}\right\|_{E}<\delta / 2$ for $k \geqslant k_{0}$ and putting $M=2 \tilde{M} / \delta$, we conclude the proof.

Denote by $\Phi$ the extremal function of a compact set $E$ in $C^{n}$, i.e.

$$
\begin{gathered}
\Phi(z, E)=\sup _{\nu \geqslant 1}\left\{\operatorname { s u p } \left\{\left|P_{\nu}(z)\right|^{1 / v}: P_{\nu} \text { is a polynomial in } z=\left(z_{1}, \ldots, z_{n}\right)\right.\right. \\
\text { such that } \left.\left.\operatorname{deg} P_{\nu} \leqslant \nu \text { and }\left\|P_{\nu}\right\|_{E} \leqslant 1\right\}\right\}, \quad z \epsilon C^{n},
\end{gathered}
$$

introduced by Siciak [5]. We shall often use the following properties of $\Phi(z, E)$ (see [5]):

$$
\begin{gathered}
\text { (1) } \Phi(z, E) \geqslant 1, \quad z \epsilon C^{n} \quad \text { and } \quad \Phi(z, E)=1, \quad z \epsilon E, \\
\text { (2) }|P(z)| \leqslant\|P\|_{E}[\Phi(z, E)]^{\operatorname{deg} P,} \quad z \epsilon C^{n} \text {, for every polynomial } P, \\
\text { (3) } \Phi(z, E) \leqslant \Phi(z, F), \quad z \epsilon C^{n}, \text { if } F \subset E, \\
\text { (4) } \quad \Phi(z, E)=\max _{1 \leqslant i \leqslant n}\left\{\Phi\left(z_{i}, E_{i}\right)\right\}, \quad z \epsilon C^{n} \text {, for } E=E_{1} \times \ldots \times E_{n} .
\end{gathered}
$$

We add that in the case $n=1$ the function $\Phi(z, E)$ is equivalent to Leja's extremal function $L=L(z, E)$ (see [3]). This note and properties (3), (4) give us some criteria for the continuity of $\Phi(z, E), E \subset C^{n}$, expressed by properties of $L$.

The results of the previous lemmas enable us to prove the following

THEOREM 1. Let $E$ be a compact set in $C^{n}$. Let $f \in \mathscr{B}\left(E,\left[\left\{v_{k}\right\}\right]\right)$ and let $\varphi$ be a holomorphic function in an open set $\Omega$ in $C$ such that $F=f(E) \subset \Omega$.

$1^{\circ}$ If $E=\hat{E}$ and the following condition is satisfied:

Condition (W). There exist polynomials $\left\{P_{\mu_{k}}\right\}, \operatorname{deg} P_{\mu_{k}} \leqslant \mu_{k},\left\{\mu_{k}\right\} \in\left[\left\{v_{k}\right\}\right]$, a neighbourhood $U$ of $E$ in $C^{n}$ and constants $A>0$ and $k_{0}>0$ such that

$$
\left\|f-P_{\mu_{k}}\right\|_{E} \leqslant M \varrho^{\mu_{k}} \quad \text { for } k \geqslant 1,
$$

$M$ and $\varrho$ being constants independent of $k, \varrho \epsilon(0,1)$,

$$
P_{\mu_{k}}(U) \subset \Omega, \quad k \geqslant k_{0},
$$

and

$$
\sup _{\boldsymbol{z} \in U}\left|\varphi\left(\boldsymbol{P}_{\boldsymbol{\mu}_{k}}(z)\right)\right| \leqslant A^{\mu_{k}} \quad \text { for } k \geqslant k_{0},
$$

then $\varphi \circ f \in \mathscr{B}\left(E,\left[\left\{v_{k}\right\}\right]\right)$.

$2^{\circ}$ If the extremal function $\Phi(z, E)$ is continuous in $E$ and $\varphi$ is a rational function, then Condition (W) is necessary that $\varphi \circ f_{\epsilon} \mathscr{B}\left(E,\left[\left\{v_{k}\right\}\right]\right)$.

Proof. $1^{\circ}$ By Condition (W) and Lemma 1, for every $k \geqslant k_{0}$ there exist polynomials $\left\{R_{\mu}^{k}\right\}$ such that

$$
\left\|\varphi \circ P_{\mu_{k}}-R_{\mu}^{k}\right\|_{E} \leqslant M_{1} A^{\mu_{k} \cdot \varrho_{1}^{\mu}} \quad \text { for } \mu=1,2, \ldots,
$$


$M_{1}$ and $\varrho_{1}$ being constants independent of $k, \varrho_{1} \epsilon(0,1)$. Take an integer $l$ so large that $A \varrho_{1}^{l} \leqslant \varrho_{1}$. Then, by (1), we obtain

$$
\left\|\varphi \circ P_{\mu_{k}}-R_{l \mu_{k}}^{k}\right\|_{E} \leqslant M_{1} \varrho_{1}^{\mu_{k}} \quad \text { for } k \geqslant k_{0} .
$$

On the other hand, by (i) of Condition (W) and Lemma 5, we have

$$
\left\|\varphi \circ f-\varphi \circ P_{\mu_{k}}\right\|_{E} \leqslant M_{2} \varrho^{\mu_{k}}, \quad k \geqslant k_{1},
$$

where the constant $M_{2}$ does not depend on $k$. By (2), (3) and the triangle inequality we get

$$
\left\|\varphi \circ f-R_{l \mu_{k}}^{k}\right\|_{E} \leqslant M \eta^{l \mu_{k}},
$$

where $\eta=\left[\max \left(\varrho, \varrho_{1}\right)\right]^{1 / l}$ and $M=\max \left(M_{1}, M_{2}\right)$. This implies that $\varphi \circ f_{\epsilon} \mathscr{B}\left(E,\left[\left\{v_{k}\right\}\right]\right)$.

$2^{\circ}$ Suppose that $f, \varphi \circ f \in \mathscr{B}\left(E,\left[\left\{v_{k}\right\}\right]\right)$, where

$$
\varphi(w)=\frac{W_{p}(w)}{Z_{q}(w)}=\frac{a\left(w-a_{1}\right) \ldots\left(w-a_{p}\right)}{b\left(w-\beta_{1}\right) \ldots\left(w-\beta_{q}\right)},
$$

$\alpha_{i} \neq \beta_{j}, i=1, \ldots, p, j=1, \ldots, q, \beta_{j} \epsilon C \backslash F$. By our assumptions there exist polynomials $\left\{P_{\mu_{k}}\right\},\left\{Q_{\omega_{k}}\right\}$, where $\left\{\mu_{k}\right\},\left\{\omega_{k}\right\} \in\left[\left\{v_{k}\right\}\right], \operatorname{deg} P_{\mu_{k}} \leqslant \mu_{k}$, $\operatorname{deg} Q_{\omega_{k}} \leqslant \omega_{k}$, and constants $M$, $\varrho$ independent of $k, \varrho \epsilon(0,1)$, such that

$$
\left\|f-P_{\mu_{k}}\right\|_{E} \leqslant M \varrho^{\mu_{k}} \quad \text { for } k \geqslant 1,
$$

and

$$
\left\|\varphi \circ f-Q_{\omega_{k}}\right\|_{E} \leqslant M \varrho^{\omega_{k}} \quad \text { for } k \geqslant 1 .
$$

By the definition of $\mathscr{B}\left(E,\left[\left\{v_{k}\right\}\right]\right)$ we may assume that $\mu_{k}=\omega_{k}=v_{k}$, $k \geqslant 1$. It follows from (4) and Lemma 5 that

$$
\left\|\varphi \circ f-\varphi \circ P_{v_{k}}\right\|_{E} \leqslant M_{1} \varrho^{v k} .
$$

Hence and by (5), we have

$$
\left\|W_{p} \circ P_{v_{k}}-\left(Z_{q} \circ P_{v_{k}}\right) Q_{v_{k}}\right\|_{E} \leqslant M_{1}\left\|Z_{q} \circ P_{v_{k}}\right\|_{E} e^{v_{k}} .
$$

By (3) and Lemma 5 there exists a constant $M_{2}$ such that

$$
\left\|Z_{q} \circ P_{\nu_{k}}\right\|_{E} \leqslant M_{2} \quad \text { for } k \geqslant 1 .
$$

Hence by (6) and property (2) of the extremal function $\Phi(z, E)$ we obtain

$$
\left|W_{p}\left(P_{v_{k}}(z)\right)-Z_{q}\left(P_{v_{k}}(z)\right) Q_{v_{k}}(z)\right| \leqslant M_{3} \varrho^{v_{k}}[\Phi(z, E)]^{r v k},
$$

for $z \epsilon C^{n}$, where $M_{3}=M_{1} \cdot M_{2}, r=\max (p, q+1)$. Take a number $\eta \epsilon(\varrho, 1)$. Since $\Phi(z, E)$ is continuous in $E$ and because of property (1) of $\Phi$, there 
exists an open set $U$ such that $E \subset U$ and $\varrho \Phi^{r}(z, E)<\eta$ for $z \epsilon U$. Hence by (7) we get

$$
\left|W_{p}\left(P_{v_{k}}(z)\right)-Z_{q}\left(P_{v_{k}}(z)\right) Q_{v_{k}}(z)\right| \leqslant M_{\mathrm{s}} \eta^{v_{k}} \quad \text { for } z \epsilon U .
$$

Suppose that $Z_{q}\left(P_{v_{k}}(\dot{z})\right)=0$ for a point $\dot{z} \in U$. Then $P_{v_{k}}(\dot{z})=\beta_{j_{0}}$ for a certain $j_{0} \in\{1, \ldots, q\}$. Write $\delta=\min _{i, j}\left\{\left|\alpha_{i}-\beta_{j}\right|\right\}$. By (8) we would have

$$
0<|b| \delta^{p} \leqslant\left|b\left(\beta_{j_{0}}-\dot{a}_{1}\right) \ldots\left(\beta_{j_{0}}-a_{p}\right)\right|=\left|W_{p}\left(\beta_{j_{0}}\right)\right| \leqslant M_{3} \eta^{\nu_{k}} .
$$

This is impossible for sufficiently large $k$. So, we have

$$
Z_{q}\left(P_{v_{k}}(z)\right) \neq 0 \quad \text { for } z \in U, k \geqslant k_{0}=k_{0}(\delta, p) .
$$

This means that (ii) of Condition $(W)$ is satisfied.

It remains to prove (iii). To this end take $\varepsilon=\frac{1}{2} \min _{1 \leq j \leq q}\left\{\operatorname{dist}\left(F, \beta_{j}\right)\right\}$ and write $F_{\varepsilon}=\{w \in O: \operatorname{dist}(w, F)<\varepsilon\}$. By $(4)$,

Hence

$$
P_{v_{k}}(z) \in F_{\varepsilon} \quad \text { for } z \in E \text {, if } k \geqslant k_{1}=k_{1}(\varepsilon) \text {. }
$$

$$
\min _{z \in E}\left|Z_{q}\left(P_{v_{k}}(z)\right)\right| \geqslant \inf _{w \in F_{s}}\left|Z_{q}(w)\right|=m>0 \quad \text { for } k \geqslant k_{2},
$$

where $k_{2}=\max \left(k_{0}, k_{1}\right)$. By (9), (10) and Lemma 4 there exists an open set $V$ such that $E \subset V \subset U$ and

$$
\left|Z_{q}\left(P_{v_{k}}(z)\right)\right| 2^{q v_{k}} \geqslant m \quad \text { for } z \in V, k \geqslant k_{2} .
$$

Hence, by (4), Lemma 5 and property (2) of $\Phi$, we obtain

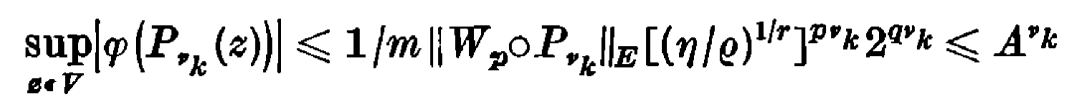

for $k \geqslant k_{2}$, where $A$ is a suitably chosen constant independent of $k$. The proof is completed.

Remark. If $E \neq \hat{E}$, then, in general, Condition (W) is not sufficient for $\varphi \circ f_{\epsilon} \mathscr{B}\left(E,\left[\left\{v_{k}\right\}\right]\right)$. One can easily come to this conclusion by considering $E=\{z \epsilon C:|z|=1\}, f(z)=z$ and $\varphi(w)=1 / w$.

Nevertheless, because of Lemma 3 , given $f \in \mathscr{B}\left(E,\left[\left\{v_{k}\right\}\right]\right)$, we may always assume that $E=\hat{E}$.

TheOREM 2. Let $E$ and $\varphi$ be the same as in $2^{\circ}$ of Theorem 1. If Condition (W) is satisfied for polynomials $\left\{\boldsymbol{P}_{\mu_{k}}\right\}$, then it is satisfied for every sequence of polynomials $\left\{Q_{x_{k}}\right\}$ such that $\left\{x_{k}\right\} \in\left[\left\{\mu_{k}\right\}\right], \operatorname{deg} Q_{x_{k}} \leqslant x_{k}$ and

$$
\left\|f-Q_{x_{k}}\right\|_{E} \leqslant M_{1} \varrho_{1}^{\alpha_{k}}, \quad k \geqslant 1
$$

$M_{1}$ and $\varrho_{1}$ being constants independent of $k, \varrho_{1} \epsilon(0,1)$.

Proof. We have only to prove (ii) and (iii) of Condition (W) for $\left\{Q_{x_{k}}\right\}$. 
(ii) If $\Omega=C$, the proof is trivial. Assume that $C \backslash \Omega=B \neq \emptyset$. By (1), (i) of Condition (W) for $\left\{P_{\mu_{k}}\right\}$ and the definition of $\left[\left\{\mu_{k}\right\}\right]$ we have

$$
\left\|P_{\mu_{k}}-Q_{x_{k}}\right\|_{E} \leqslant M_{2} \varrho_{2}^{\omega_{k}}, \quad k \geqslant 1,
$$

for suitable constants $M_{2}$ and $\varrho_{2}, \varrho_{2} \in(0,1), \omega_{k}=\max \left(\mu_{k}, x_{k}\right)$. Take a number $\eta \in\left(\varrho_{2}, 1\right)$. Since the extremal function $\Phi(z, E)$ is continuous in $E$, there exists an open set $\nabla, E \subset \nabla$, such that $\varrho_{2} \Phi(z, E) \leqslant \eta$ for $z \epsilon \operatorname{cl} V$. Hence, by (2) and property (2) of $\Phi$, we get

$$
\left|P_{\mu_{k}}(z)-Q_{x_{k}}(z)\right| \leqslant M_{2} \eta^{\omega_{k}} \quad \text { for } z \in V, k \geqslant 1 .
$$

Write $2 m=\operatorname{dist}(F, B)$. By (i) of Condition (W) for every point $w \in B$ we have

$$
\left|P_{\mu_{k}}(z)-w\right| \geqslant|f(z)-w|-\left|f(z)-P_{\mu_{k}}(z)\right| \geqslant 2 m-M Q^{\mu_{k}} \geqslant m>0
$$

for $z \epsilon E$ and $k \geqslant k_{1}=k_{1}(m)$. Hence, by (ii) of Condition (W), the family $\mathscr{F}=\left\{P_{\mu_{k}}-w: w \in B, k \geqslant k_{2}=\max \left(k_{0}, k_{1}\right)\right\}$ of polynomials in $z$ satisfies the assumptions of Lemma 4 . So, for every $\lambda>1$ we can find an open set $V_{\lambda}$ such that $E \subset V_{\lambda} \subset U$ and

$$
\left|P_{\mu_{k}}(z)-w\right| \lambda^{\mu_{k}} \geqslant m, \quad z \in V_{\lambda}, w \in B, k \geqslant k_{2} .
$$

If we take $\lambda<1 / \eta$, then, by (3) and (4), we get

$$
\left|Q_{\boldsymbol{x}_{k}}(z)-w\right| \geqslant\left|P_{\mu_{k}}(z)-w\right|-\left|P_{\mu_{k}}(z)-Q_{x_{k}}(z)\right| \geqslant m \lambda^{-\mu_{k}}-M_{2} \eta^{\omega_{k}}>0
$$

for $z \in V_{\lambda} \cap V, w \in B, k \geqslant k_{3}$, where $k_{3}$ is sufficiently large. Thus, $Q_{x_{k}}\left(V_{\lambda} \cap V\right)$ $\subset \Omega$ for $k \geqslant k_{\mathrm{a}}$ as asserted.

(iii) Since the function $\varphi$ is rational, it is enough to apply property (2) of the extremal function $\Phi$ and Lemma 4 .

Now let $E$ be a polynomially convex compact set in $C\left(^{2}\right)$ and let $f \in \mathscr{B}\left(E,\left[\left\{v_{k}\right\}\right]\right)$. Assume that the extremal function $\Phi(z, E)$ is continuous in $E$. Comparing Lemma 2 and Theorem 1, by the Montel theorem, gives the following

THEOREM 3. If $\varphi$ is a rational function with at least two poles lying in $C \backslash f(E)$, then $\varphi \circ f \epsilon \mathscr{B}\left(E,\left[\left\{v_{k}\right\}\right]\right)$ if and only if there exists a function $\tilde{f}$ holomorphic in a neighbourhood of $E$ such that $\left.\tilde{f}\right|_{E}=f$.

We shall now illustrate our results by means of some examples.

Counter-eXaMPLE 1 . Let $E=\{z \in C:|z| \leqslant 1\}$. Then $\Phi(z, E)$ $=\max \{|z|, 1\}$ for $z \epsilon C$ (see [5]). Take a sequence $\left\{v_{k}\right\}$ of positive integers such that $v_{k+1} / v_{k} \rightarrow \infty$ as $k \rightarrow \infty$ and define

$$
f(z)=\sum_{k=1}^{\infty} \frac{z^{y k}}{a^{k k-1}} \quad(a>1) .
$$

(ग) In this case it is well known that $E=\hat{E}$ if and only if the set $O \backslash E$ is connected.

6 - Annales Polonic1 Mathematic1 XXVI 
One can easily check that $f \epsilon \mathscr{B}\left(E,\left\{v_{k}\right\}\right)$ and $f$ cannot be analytically continued onto any neighbourhood of $E$. Let $\varphi$ be a rational function with at least two poles. Then it follows from Theorem 3 that $\varphi$ of $\not \mathscr{B}(E$, $\left.\left[\left\{v_{k}\right\}\right]\right)$.

Counter-example 2. Define $E$ and $f$ as above and set

$$
g(z)=f^{2}(z)-M^{2},
$$

where $\|f\|_{E}<M$. Since $\mathscr{B}\left(E,\left[\left\{v_{k}\right\}\right]\right)$ is a ring; then $g \in \mathscr{B}\left(E\right.$, $\left.\left[\left\{v_{k}\right\}\right]\right)$. Because of Theorem 3 it is seen that $1 / g \notin \mathscr{B}\left(E,\left[\left\{v_{k}\right\}\right]\right)$.

Counter-example 3. Define the sequence $\left\{v_{k}\right\}$ as

$$
v_{0}=1, \quad v_{k+1}=2^{v_{k}}, \quad k \geqslant 0,
$$

and set

$$
f(x)=\sum_{l=0}^{\infty} \frac{\cos \nu_{k} \arccos x}{v_{k}} \quad \text { for } x \in E=[-1,1] .
$$

It is known (Bernstein [1], p. 294) that $f \in \mathscr{B}\left(E,\left\{v_{k}\right\}\right)$ and $f$ is not differentiable in $E$. Define

$$
g(x)=f(x)+M,
$$

where $\|f\|_{E}<M$. We will show that $1 / g \notin \mathscr{B}\left(E,\left[\left\{v_{k}\right\}\right]\right)$. To this end write

$$
P_{v_{k}}(z)=M+\sum_{l=0}^{k} \frac{\cos v_{l} \arccos z}{v_{l}}=M+\sum_{l=0}^{k} \frac{\left(z+\sqrt{z^{2}-1}\right)^{p} l+\left(z-\sqrt{z^{2}-1}\right)^{v_{l}}}{2 v_{l}} .
$$

It is clear that $P_{v_{k}}$ is a polynomial in $z$ of degree $v_{k}$. Moreover,

$$
\left\|g-P_{v_{k}}\right\|_{E}<2(1 / 2)^{v^{k}} .
$$

Fix a number $r>1$. Since the sequence $\left\{v_{k} / v_{k-1}\right\}$ is increasing, for $\left|z+\sqrt{z^{2}-1}\right|=R \geqslant r$ we have

$$
\begin{aligned}
& \left|P_{v_{k}}(z)\right| \geqslant\left|\frac{\left(z+\sqrt{z^{2}-1}\right)^{v^{k} k}+\left(z-\sqrt{z^{2}-1}\right)^{\nu} k}{2 v_{k}}\right|- \\
& -\left|M+\sum_{l=0}^{k-1} \frac{\left(z+\sqrt{z^{2}-1}\right)^{\nu_{l}}+\left(z-\sqrt{z^{2}-1}\right)^{\nu_{l}}}{2 v_{l}}\right| \\
& \geqslant \frac{R^{v_{k}}-R^{-v_{k}}}{2 v_{k}}-\sum_{l=0}^{k-1} \frac{R^{p_{l}}+R^{-v_{l}}}{2 v_{l}}-M \\
& >\frac{1}{2}\left[\left(\frac{R^{v} k^{/ \nu} k-1}{2}\right)^{v_{k-1}}-\sum_{l=1}^{k-1}\left(\frac{R^{v} k^{/ v} k-1}{2}\right)^{v_{l-1}}\right]-\left(M+\frac{R}{2}+1\right)>0 \text {, }
\end{aligned}
$$


as $k \geqslant k_{0}=k_{0}(r)$. Thus, all the zeros of the polynomials $\left\{P_{v_{k}}\right\}\left(k \geqslant k_{0}\right)$ are contained in the ellipse $\left\{z \epsilon C:\left|z+\sqrt{z^{2}-1}\right|<r\right\}$ with the foci -1 and 1. So, the sequence $\left\{P_{v_{k}}\right\}$ does not satisfy (ii) of Condition (W) for $\varphi(w)=1 / w$. Since $\Phi(z,[-1,1])=\left|z+\sqrt{z^{2}-1}\right|$ for $z \epsilon C$ (see [3]), it is continuous in $C$. Hence, by Theorems 1 and $2,1 / g \notin \mathscr{B}\left([-1,1],\left[\left\{v_{k}\right\}\right]\right)$.

EXAMPLE 4. Let $E$ be a compact set in $C^{n}$. If $f \in \mathscr{B}\left(E,\left[\left\{v_{k}\right\}\right]\right)$ and $\varphi$ is a polynomial; then $\varphi \circ f_{\epsilon} \mathscr{B}\left(E,\left[\left\{\nu_{k}\right\}\right]\right)$.

EXAMPLE 5. Let $E$ be a polynomially convex compact set in $C^{n}$ such that the extremal function $\Phi(z, E)$ is continuous in $E$. Take an increasing sequence $\left\{v_{k}\right\}$ of positive integers such that $\nu_{k+1} / R^{v_{k}} \rightarrow \infty$ as $k \rightarrow \infty$ for a certain constant $R>1$. Write $\mu_{k}=\left[R^{\nu} k\right], k=1,2, \ldots$ Given an $\varrho \epsilon(0,1)$, we put

$$
\varepsilon_{v}=e^{\mu} \quad \text { for } v_{k} \leqslant \nu<\nu_{k+1}, k \geqslant 1 .
$$

By the well-known Bernstein theorem (see [6]) there exists a function $f \in \mathscr{C}(E)$ such that

Since

$$
\mathscr{E}_{v}(f, E)=\varepsilon_{v}, \quad \nu=1,2, \ldots
$$

$$
\limsup _{v \rightarrow \infty} \sqrt{\mathscr{E}_{\nu}(f, \boldsymbol{E})} \geqslant \lim _{k \rightarrow \infty} \varrho^{\mu_{\nu} / \nu} k+1^{-1}=1 ;
$$

then, in virtue of Lemma 1 (for the case $f_{k}=f$ ), the function $f$ cannot be continued to a holomorphic function onto any neighbourhood of $E$. Take polynomials $P_{v_{k}}$ such that

$$
\mathscr{E}_{v_{k}}(f, E)=\left\|f-P_{v_{k}}\right\|_{E}, \quad k \geqslant 1 .
$$

Since $\mathscr{E}_{\mu}(f, E) \leqslant \mathscr{E}_{v}(f, E)$ for $\mu \geqslant \nu$, then $f \in \mathscr{B}\left(E,\left\{\mu_{k}\right\}\right)$. By the assumption of continuity of $\Phi(z, E)$ in $E$ we can choose an open set $U$, $E \subset U$, such that $\Phi(z, E)<R$ for $z \epsilon U$. Then, by property (2) of $\Phi$, we have

and

$$
\left|e^{P_{y}}(z)\right| \leqslant A^{R^{y} k}, \quad z \in U
$$

$$
\left|e^{-P_{v_{k}}}(z)\right| \leqslant A^{R^{\nu} k}, \quad z \in U,
$$

$A$ being a positive constant. Hence the sequences $P_{\mu_{k}} \equiv P_{v_{k}}$ and $Q_{\mu_{k}} \equiv-P_{v_{k}}$ $(k=1,2, \ldots)$ satisfy Condition (W) for $\varphi(w)=e^{w}$. Thus, by Theorem 1 , the functions $e^{f}$ and $e^{-f}$ are members of $\mathscr{B}\left(E,\left[\left\{\mu_{k}\right\}\right]\right)$.

\section{References}

[1] С. Н. Бернштени, Собрание сочинений, Иадат. АН СССР, т. 1, 1952.

[2] F. Leja, Sur une propriété des suites de polynomes, Ann. Soc. Polon. Math. 21 (1948). 
[3] - Theory of analytic functions, Warszawa 1957 (Polish).

[4] W. Pleśniak, Quasianalytic functions of several complex variables, Zesz. Nauk. UJ 15 (1971), p. 135-145.

[5] J. Siciak, On some extremal functions and their applications in the theory of analytic functions of several complex variables, Trans. Amer. Math. Soc. 105 (2) (1962), p. 322-357.

[6] А. Ф. Ти ман, Теория приближения функұий дейстөительного переменного, Москва 1960.

Reģu par la Rédaction le 4. 2. 1971 